\title{
Evaluating the Impact of Thermal Noise on AODV, DSDV and DSR Routing Protocols in Mobile Ad-hoc Networks
}

\author{
Mahsa Paknezhad \\ Department of Computer Engineering and IT \\ Shiraz University of Technology \\ Modarress Blvd. 71555-313 \\ Shiraz, Fars, Iran
}

\author{
Manijeh Keshtgari, PhD. \\ Department of Computer Engineering and IT \\ Shiraz University of Technology \\ Modarress Blvd. 71555-313 \\ Shiraz, Fars, Iran
}

\begin{abstract}
Because of their good support and easy deployment, wireless ad-hoc networks are currently widely being used for many industrial or military applications. Like other communication networks, environmental conditions can have considerable impact on the performance of wireless ad-hoc networks. Temperature is a good example which results in thermal agitation of the charge carriers. Consequently, we should be able to precisely estimate the performance of these networks in various environmental conditions to see whether they satisfy our expectations or not before any deployment. Investigating the effect of Thermal noise on the routing performance of Wireless Ad-hoc Networks is something never done before. This paper evaluates the impact of Thermal noise on the routing capability of Mobile Ad-hoc Networks (MANETs); which are a kind of Wireless ad-hoc Networks; in existence of thermal noise. The results can be a useful guidance for outdoor implementation of MANETs for industrial process automation and control applications.
\end{abstract}

\section{General Terms}

Performance Evaluation of Routing Algorithms

\section{Keywords}

Thermal noise; temperature; Wireless Ad-hoc Networks; adhoc routing protocols; industrial process automation applications; control applications; NS2 Simulator.

\section{INTRODUCTION}

Thermal noise also named Johnson-Nyquist noise contributes to the vibration of charge carriers. This noise; like other forms of noise; is random in nature, so it is not possible to predict its impact on the waveform in order to reduce the effects by cancellation [1]. Many experiments have been carried out so as to analyze the impact of Thermal noise on a vast variety of networks and devices, such as Single Flux Quantum devices [2], Fiber-To-The-Home (FTTH) customer access networks [3], Ultra-High-Speed High Temperature Super-conductor digital circuits [4] etc., which we will review in the next section. These experiments reveal that temperature has a direct impact on communications between wireless nodes. It is proved that "less transmission power is required at low temperatures" [5] and that although temperature has a negligible effect on path propagation, radio equipment is highly sensitive to thermal noise [6]. A considerable number of experiments have been conducted to compare the performance of well-known routing protocols AODV, DSDV and DSR [7, 8, 9], but evaluating temperature effect on these ad-hoc routing protocols has rarely been investigated. In this paper, we will assess the behavior of three different ad-hoc routing protocols, namely, AODV, DSDV and DSR in existence of Thermal noise. We will utilize NS2 simulator [10] to simulate Thermal noise and compare the performance of the three above routing protocols.

This paper is structured as follows. In the next section, we will provide a brief review of other projects carried out with the purpose of investigating the impact of thermal noise on network communications and devices. In section 3, we will present our own approach for investigating and comparing temperature effect on the performance of ad-hoc routing protocols. In section 4, we will show the results of our simulations. Finally, a summery and conclusion are givenin Section 5 .

\section{RELATED WORK}

In the following, we will review some experiments conducted to evaluate the impact of Thermal noise on communication networks and devices.

Ab-Rahman et al. [3] studied Passive Optical Networks (PON, These networks "bring optical fiber cabling all the way or most of the way to the end users" [6]) in terms of Thermal noise effects and came to multiple important conclusions. They showed that by reducing the thermal noise in the network, we can achieve a good improvement in the BER. Moreover, this experiment proved that since sensitivity is inversely proportional to the level of thermal noise, for a high sensitive photo detector thermal noise has to be reduced. They also showed that for the same sensitivity, different rates of transmission will result in different thermal noise values.

Boano et al. [5] used a sensor net as their case study and investigated temperature effect on low-power wireless communications. They showed that high temperature may cause loss of connectivity in the network and that as the temperature decreases so does the RSSI (Received Signal Strength Indicator) noise floor. To sum up, they revealed that temperature has a significant impact on signal strength and link quality, so they suggested considering the influence of temperature variations when deploying a wireless sensor network.

Otero et al. [6] analyzed the potential impact of rain, fog, wind and temperature on the performance of network equipment in a high performance, wide area, and wireless network. Gathering information about network throughput, error rates etc. on a daily basis they revealed that "different weather phenomena affect the wireless links in a variety of ways". Their experiment showed that wind storms cause movements in the antenna and consequently make the antenna skew. Moreover, it was proved that high temperature causes the output waveform to distort and results in errors in the packet processors in the routers. 
Last but not least, Krusevac et al. [11] investigated the effect of thermal noise on a multi-antenna system with coupled antennas. Having included only two or three half-way dipoles in order to make drawing precise conclusions much easier, they showed that "for close antenna spacings with high coupling the thermal noise power of one dipole in two- or three-dipole arrays decreases". In general, a partial correlation between thermal noise and antenna spacing below a certain wavelength was proved to exist.

In this paper, we study the effect of thermal noise on network communications in a temperature range in which network devices are designed to operate well. Consequently, we will not consider the impact of temperature on these devices.

\section{IMPLEMENT THERMAL NOISE INNS2}

In this section, we will present how to implement Thermal Noise in NS2. First, we have to create C++ files "ThermalError.cc" and "ThermalError.h". For this purpose, NS2 built-in class ErrorModel implemented in "errmodel.cc" and "errmodel.h" is utilized. You can find these two files in ns-allinone package or you can visit [13]. This class simulates link-level errors or loss by using the error_flag of the packet's common header. It defines unit and ranvar to specify the unit of error and the random variable for generating errors respectively [12].

However, "ErrorModel only implements a simple policy based on a single error rate" [12]. We extend this class to implement our own ThermalError class. The structure of our ThermalError class can be seen in figure 1 .

\begin{tabular}{|l|}
\hline ThermalError.h \\
\hline \hline Class ThermalError: public ErrorModel \{ \\
public: \\
ThermalError(); \\
virtual void setRate_(); \\
protected: \\
Int command(int argc, const char*const*argv); \\
double temp_; \\
int tech_; \\
double m_; \\
int modu_; \\
\};
\end{tabular}

Figure 1. The Structure of Thermal Error Class

The function setRate_ is used to set value to the variable rate of superclass ErrorModel. This variable defines the probability of error in the communication (BER). Other variables in the ThermalError class are discussed later in this section. Now, we need to define the relation between Thermal Noise and BER. Thermal noise power at temperature $\mathrm{T}$ and bandwidth $B_{w}$ is given as:

$$
N=K T B_{w}
$$

Where $\mathrm{K}$ is the Boltzmann's constant $1.3803 \times 10^{-23}(\mathrm{~J} / \mathrm{K})$ and $\mathrm{T}$ is temperature in Kelvin. In order to define noise power in $\mathrm{dBW} / \mathrm{Hz}$ the above equation changes to:

$$
N=-228.6 d B W+10 \log T+10 \log B_{w}
$$

Moreover, the following equation provides an approximation to error probability $\left(\mathrm{P}_{\mathrm{b}}\right)$ in communications with high SNRs:

$$
P_{b} \approx \frac{M-1}{2} \operatorname{erfc}\left(\sqrt{\gamma \frac{E_{b}}{N}}\right)
$$

In this equation, $\mathrm{M}$ defines the number of waveforms used in the modulator and $E_{b}$ isthe energy per bit in the communication [15]. $E_{b}$ can be defined for different communication technologies by dividing carrier power by the bit rate in the communication [16]. As an example, the energy per bit of an $802.11 \mathrm{~b}$ radio is $112 \mathrm{~nJ} / \mathrm{bit}$ and for 802.15 .4 radio it is $979 \mathrm{~nJ} / \mathrm{bit}$ [17].

$\gamma$ represents the power efficiency of the modulation scheme. It is defined as $d_{\min }^{2} / 4 E_{b}$ in which $d_{\min }$ is the minimum Euclidean distance between any two elements of the modulator signal set. However, for different modulation schemes $\gamma$ can be calculated using M [15]. For instance, in a communication which utilizes PSK modulation we have:

$$
\gamma=\left(\sin \frac{\pi}{M}\right)^{2} \times \log _{2} M
$$

As a consequence, by knowing $\mathrm{M}$, modulation scheme, temperature, communication technology used (e.g. 802.11b) and the bandwidth of a communication we can measure the BER of that communication. Variables $m_{-}$, modu_, temp and tech_ in ThermalError class and bandwidth_ in the superclass ErrorModel determine these parameters of the communication respectively.

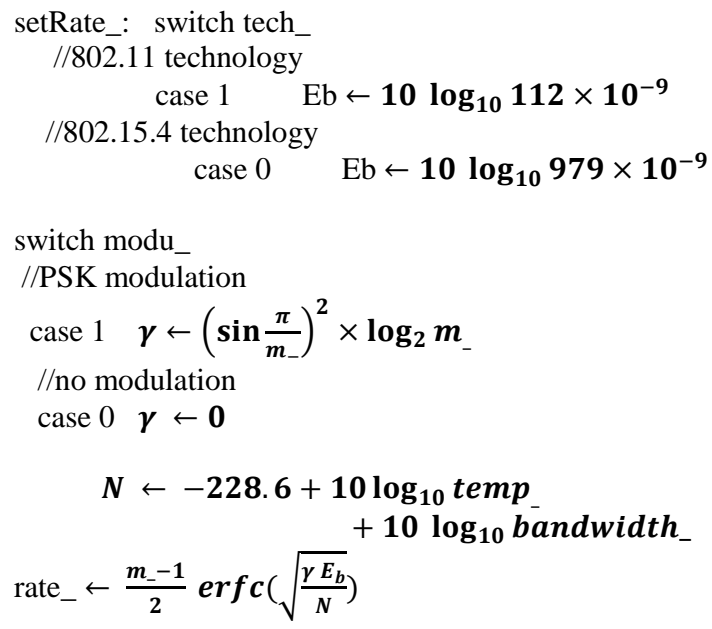

Figure 2. The Structure of setRate_Function

We will use the above relations to determine the rate of error in the communication. As a result, setRate_function will be as shown in figure 2 . 


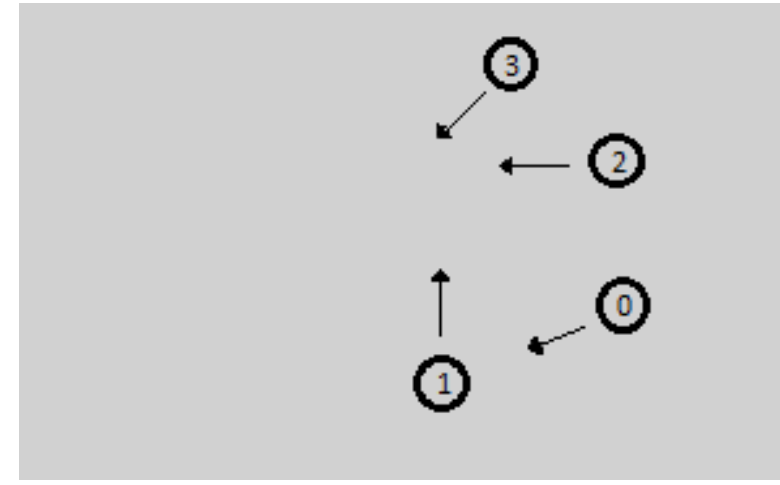

Figure 3. Initial State of our Scenario

After finishing the implementation of the ThermalError class, we should modify "ns-default.tcl" file in NS2 to include the following lines:

ErrorModel/ThermalError set temperature_ 290

ErrorModel/ThermalError set technology_ 0

ErrorModel/ThermalError set $\mathrm{m}_{-} 0$

ErrorModel/ThermalError set modulation_0

ErrorModel/ThermalError set bandwidth_ 0

This will initialize parameters tem_, tech_, $\mathrm{m}_{-}$and modu our ThermalError class respectively. Finally, we have to add "ThermalError.o" to the list of object files in "Makefile.in" file. After recompiling NS2 the ThermalError class will be ready to use.

\section{EXPERIMENTAL RESULTS}

In this section, we will use our ThermalError class to simulate

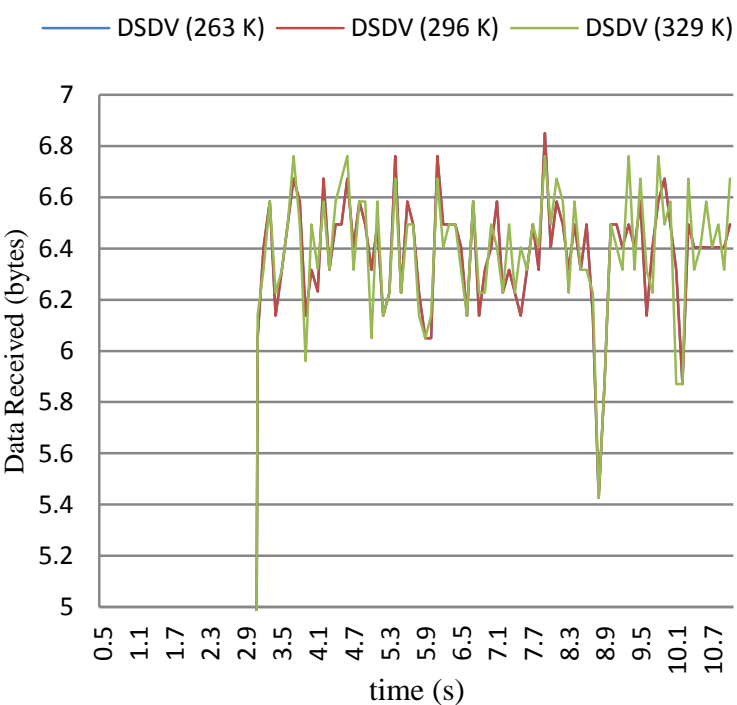

Figure 5. DSDV routing protocol

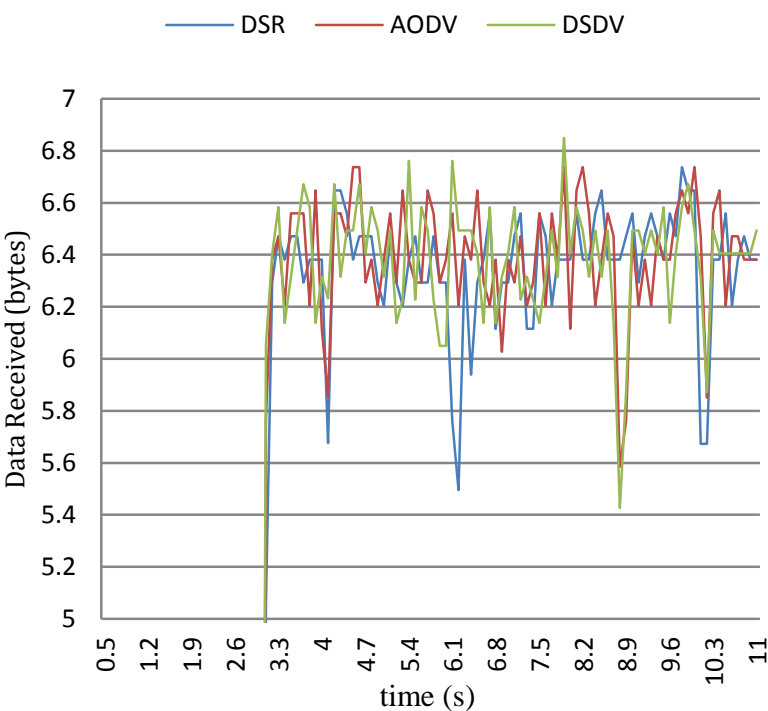

Figure 4. Data received by the four routing Protocols not considering Thermal noise

Thermal noise in scenarios for the three famous Ad-hoc routing protocols: AODV, DSDV and DSR. The scenario for all the three protocols will include four nodes moving in different directions with different speeds. The initial state of these four nodes and their moving directions are presented in figure 3 . There will be one ftp transmission between node 0 and node 1 starting at time 4.0 and ending at 11.0, and another ftp transmission between node 2 and node 3 starting at 3.0, temporarily stopping at 6.0 , again staring at 7.0 and finally ending at 10.0. First, we will run this scenario 5 times for the three routing protocols without considering Thermal noise and show the average performance of the three protocols in a diagram. Then, we will run it at three different temperatures for each routing protocol considering the existence of Thermal noise. We will measure the amount of bytes delivered at our two receivers, node 1 and node 2 at different intervals and add them together. Getting the average of the 5 runs for each routing protocol, we compare the results with each other.

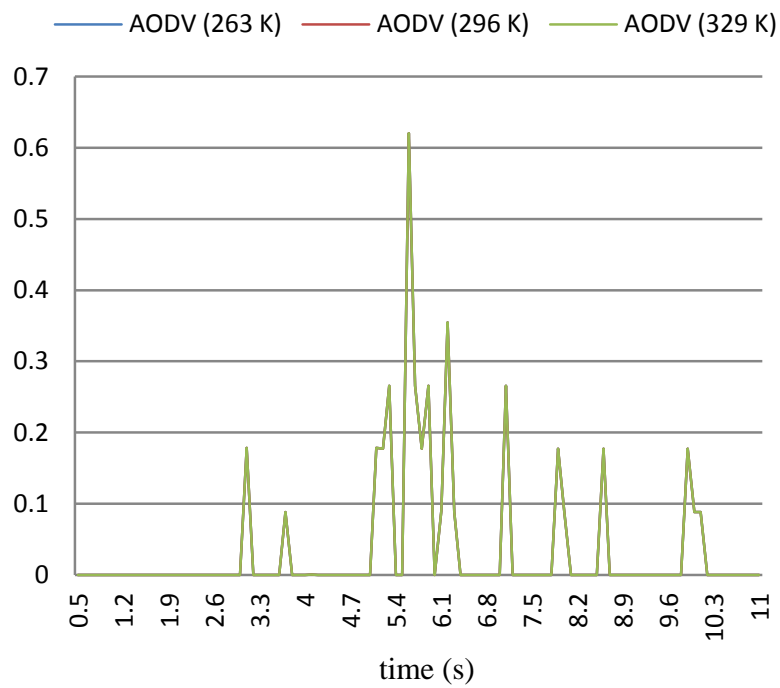

Figure 6. AODV routing protocol 
Figure 4 shows the result of running the scenario for the three routing protocols without considering Thermal noise. As the chart reveals, the performance of all the routing protocols are almost the same with very small differences. In figures 5 to 7 , we show the performance of the three routing protocols considering Thermal noise effect. Figure 5 shows the individual performance of the DSDV routing protocol at 3 different temperatures: 263 K, 296 K, and 329 K. Figures 6 and 7 show the same thing for AODV and DSR respectively. As depicted in figure 5, DSDV routing protocol performs the same at the twotemperatures: 263 kelvin and 296 kelvin. Figure 6 also reveals that the performance of AODV routing protocol is the same at the three different temperatures.

In figure 8 , the average performances of the three routing protocols are depicted. As can be seen, DSDV routing protocol significantly outperforms all the other two routing protocols. However, DSR performs very slightly better than AODV routing protocol. As the diagram shows, they perform almost the same.

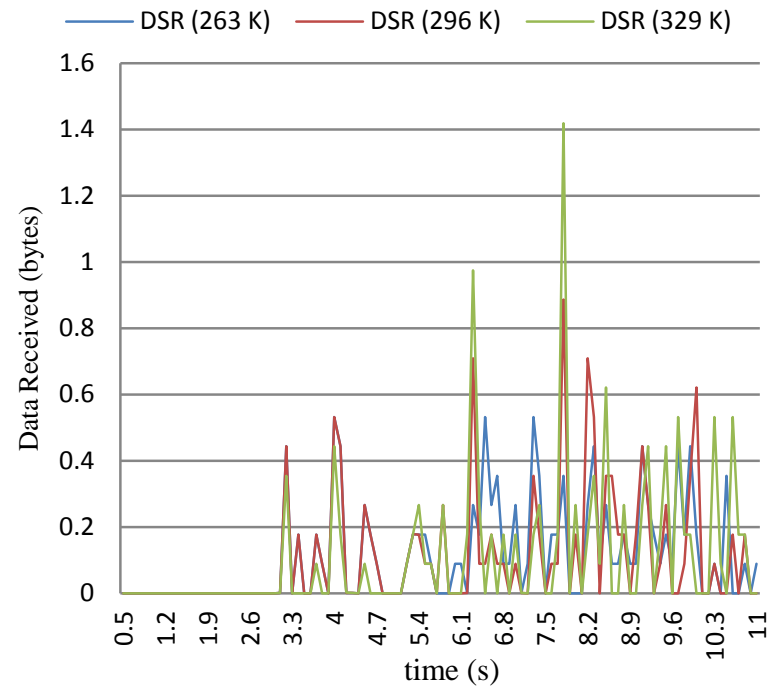

Figure 7. DSR routing protocol

In order to prove our observation, we have calculated $95 \%$ confidence interval for the sample differences of each two routing protocols. If the confidence interval includes zero we can conclude that the two routing protocols have almost the same performance, otherwise we can say with $95 \%$ confidence that the two routing protocols are significantly different in performance. As an example, we show the calculation of the $95 \%$ confidence interval for DSDV and AODV routing protocols. After calculating the mean $(\bar{x})$ of the pairwise difference of the samples of the two protocols which becomes 4.783037, we determine the standard Deviation $(\boldsymbol{\sigma})$ of the sample differences $(=2.747429)$. Since the number of our samples is 106, the $95 \%$ confidence interval of the two protocols will be as follows:

$$
\begin{gathered}
\left(4.783037-z_{0.975} \frac{2.747429}{\sqrt{106}}, 4.783037+\right. \\
\left.z_{0.975} \frac{2.747429}{\sqrt{106}}\right)=(4.516183,5.049891)
\end{gathered}
$$

Because this interval does not include zero, we can conclude with $95 \%$ confidence that DSDV is significantly better than AODV in performance. This confidence interval is also presented in table1.

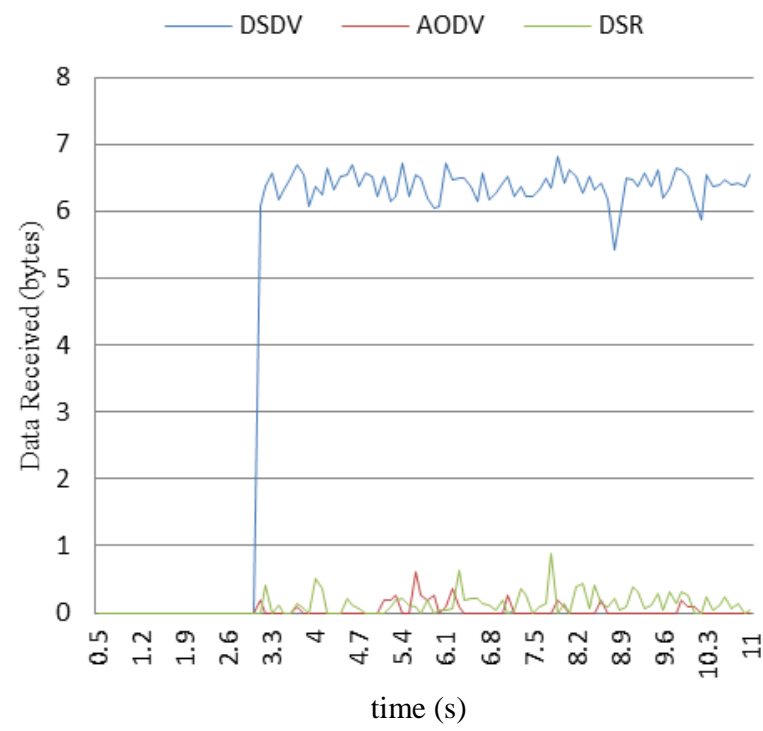

Figure 8. Data received by the four routing protocols considering Thermal noise

Table 1 shows the confidence interval for each two routing protocols. As can be easily seen, none of the specified confidence intervals include zero. As a result, we can conclude that DSDV routing protocol significantly outperforms the other two routing protocols in existence of Thermal noise, while DSR is only very slightly more efficient than AODV.

\section{CONCLUSION}

Having never been investigated in terms of Thermal noise, the performance of three different routing protocols, namely, AODV, DSDV and DSR routing protocols is revealed in this paper at different Thermal conditions. This will be a good

Table 1. Confidence interval for each two routing protocols

\begin{tabular}{|l|c|c|c|c|}
\hline Routing Protocols & Mean & Variance & Standard Deviation & Confidence Interval \\
\hline DSDV vs. AODV & 4.783037 & 7.548366 & 2.747429 & $(4.516183,5.049891)$ \\
\hline DSDV vs. DSR & 4.709713 & 7.333247 & 2.707997 & $(4.446689,4.972737)$ \\
\hline AODV vs. DSR & -0.07332 & 0.03437 & 0.185391 & $(-0.09133,-0.05532)$ \\
\hline
\end{tabular}


help for network deployment specialists to deploy networks of various applications, including industrial process automation and control applications with great efficiency. The paper shows that in existence of Thermal noise DSDV routing protocol performs much better than the other two routing protocols. DSR is the next efficient routing protocol and AODV has the poorest performance.

\section{REFERENCES}

[1] Resources and analysis for electronics engineers. [online] http://Radio-Electronics.com; owned by Adrio Communications Ltd. and edited by Ian Poole (Accessed 31 August 2012,).

[2] Kang, J. H. and Choi, S. E. 1996 Thermal-Noise-Induced Bit Error Rates of Josephson Devices, Journal of the Korean Physical Society, Vol. 30 No. 2, April 1997, pp. 306-309.

[3] Ab-Rahman, M. S., Boonchuan, Ng and Safnal, M. H. G. 2009 Thermal Noise Effect on Receiver Sensitivity in FTTH Customer Access Network -Simulation Approach, Journal of Optical Communications, J. Opt. Commun. 30 (2009), pp.95-98

[4] Jeffery, M., Xie, P. Y., Whiteley, S. R. and Van Duzer, T. 1998 Monte Carlo and Thermal Noise Analysis of Ultra-High-Speed High Temperature Superconductor Digital Circuits, presented at The Applied Superconductivity Conference, Palm Springs CA, Sept. 14-18, 1998.

[5] Boano, C. A., Tsiftes, N., Voigt, T., Brown, J. and Roedig, U., 2010 The Impact of Temperature on Outdoor Industrial Sensornet Applications, Journal of IEEE Transactions on Industrial Informatics (TII), Vol. 6 No. 3, pp. 451-459. August 2010.

[6] Otero, J., Yalamanchili, P. and Braun, H. W. 2003 High Performance Wireless Networking and Weather copyright 2005 IEEE.

[7] Lego, K., Kumar, P., Singh and Sutradher, D., 2010 Comparative Study of Adhoc Routing Protocol AODV, DSR and DSDV in Mobile Adhoc Network, Indian Journal of Computer Science and Engineering, Vol. 1 No. 4 pp.364-371.
[8] Islam, S., Hider, N., Haque, T. and miah, L. 2011 An Extensive Comparison among DSDV, DSR and AODV Protocols in MANET, International Journal of Computer Applications (0975 - 8887), Vol. 15 No.2, February 2011.

[9] Khatawkar, S. D., Pandyaji, K. K., Patil, R. M., Sali, V. M. and Kulkarni, U. L. 2012 Performance Comparison of DSDV, AODV, DSR, Routing protocols for MANETs, International Conference on Computer Networks and Communication Systems (CNCS 2012) IPCSIT vol.35 (C) (2012) IACSIT Press, Singapore.

[10] USC University of Southern California,http://www.isi.edu/nsnam/ns/(Accessed11 September 2012).

[11] Krusevac, S., Rapajic, P. B., Kennedy, R. A. and Sadeghi, P. 2005 Mutual Coupling Effect on Thermal Noise in Multi-antenna Wireless Communication.

[12] Fall, K. and Varadhan, K. The VINT Project, A Collaboration between researchers at UC Berkeley, LBL, USC/ISI, and Xerox PARC. (2007), 'The ns Manual (formerly ns Notes and Documentation)', pp. 126-130.

[13] UCLA University official website, http://www.cs.ucla.edu/classes/cs219/NS/ns-2.0/ (Accessed 18 September 2012).

[14] TechTarget (NASDAQ:TTGT) [online] http://searchtelecom.techtarget.com /definition/passiveoptical-network (Accessed 11 September 2012).

[15] Garg V. K. 2007 Wireless Communications and Networking, by Elsevier Inc. pp.106-119.

[16] Breed G. 2003 Bit Error Rate: Fundamental Concepts and Measurement Issues, from High Frequency ElectronicsCopyright copyright 2003 Summit TechnicalMedia, LLC.

[17] Lymberopoulos, D., Priyantha, N. B., Goraczko, M. and Zhao, F. 2008 Towards Energy Efficient Design of Multi-Radio Platforms for Wireless Sensor Networks. 